\title{
Blood pressure variability, prehypertension, and hypertension in adolescents
}

REVIEW

This article was published in the following Dove Press journal:

Adolescent Health, Medicine and Therapeutics

5 May 2012

Number of times this article has been viewed

\section{Donald L Batisky}

Emory Children's Center, Emory University School of Medicine, Atlanta, GA, USA
Correspondence: Donald L Batisky Emory Children's Center, 2015 Uppergate Drive NE, Atlanta, GA 30322, USA

Tel + I 4047275750

Email dbatisk@emory.edu
Abstract: Medical conditions diagnosed during adolescence may have long term impacts on the health of an individual. As a result, identifying cardiovascular risk factors earlier in life such as prehypertension (pre-HTN) and hypertension (HTN) can have significant benefits across an individual's lifespan. Diagnosing elevated blood pressure (BP) during adolescence can be difficult, partially due to the natural variability that occurs during this period of life. Levels of BP that define adolescent prehypertension/hypertension are provided as well as an abridged review of BP variability across research groups. Strategies for BP management of adolescents are considered, with the primary focus on nonpharmacologic interventions.

Keywords: HTN, pre-HTN, overweight, obesity, BP, body mass index, BMI

\section{Introduction}

Adolescence is defined as the "period between the beginning of puberty until maturity" in human development, and it extends mainly over the teen years and terminates legally when the age of majority is reached. ${ }^{1}$ The word is from the Latin root "adolescere," which means "to grow up." It is a time of rapid change in the life of an individual, and it is the time between childhood and adulthood. While children are not just little adults and adults are not just large children, adolescents are also unique and constantly changing, and during this time there are some medical problems that may surface. Elevated BP may be detected at this age, and it is important to recognize the definitions of hypertension (HTN) as well as prehypertension (pre-HTN) to appropriately counsel patients and their families about this condition.

There have been great advances in the treatment of cardiovascular disease over the last several decades, and yet the best treatment strategy continues to be the prevention of the development of the disease. Elevated BP is one of a number of modifiable risk factors for cardiovascular disease, along with dyslipidemia, cigarette smoking, diabetes, and overweight/obesity. This article will focus on HTN, and definitions of HTN and pre-HTN will be discussed first. Following those definitions, the issue of BP variability as a challenge in the management of this condition will be addressed. The various strategies for treatment will also be considered, with careful attention being paid to therapeutic lifestyle changes (TLC), also known as nonpharmacologic management, and then some guidelines for pharmacologic management will also be outlined. 


\section{Definition of prehypertension and hypertension in adolescents}

The Fourth Report on Blood Pressure in Children and Adolescents (Fourth Report) provides guidance on the diagnosis, evaluation, and management of high $\mathrm{BP}$ in this age group. The current definition of HTN is BP values that fall above the 95th percentile for age, gender, and stature on at least three occasions. This definition of HTN is one that is based on normative distribution of BP in healthy children and adolescents, and it is stratified by age, gender, and stature. ${ }^{3}$ While there are outcome data in adults that allow for a definition of HTN to be BP level above 140/90 mm Hg, similar data in adolescents do not exist. Therefore, the definition of HTN is different in adolescents, and it will depend upon the age of the adolescent. In the adolescent younger than 18 years of age, the definition of HTN is BP values that fall above the 95th percentile for age, gender, and stature on at least three occasions. ${ }^{4}$

In addition to defining $\mathrm{HTN}$ as BP being $>95$ th percentile, the National High Blood Pressure Education Program has more recently gone to staging HTN in this way: Stage 1 HTN is BP from the 95th percentile to the 99th percentile plus $5 \mathrm{~mm} \mathrm{Hg}$, and Stage $2 \mathrm{HTN}$ is BP above the 99th percentile plus $5 \mathrm{~mm} \mathrm{Hg.}{ }^{3}$ While there is no direct evidence linking the stratifications of pre-HTN, Stage 1, or Stage 2 HTN with specific outcomes in pediatric age groups, it is felt that pediatric patients with Stage 2 HTN (higher relative readings for age, gender and stature) are more likely to have secondary forms of HTN. There seems to be a general feeling that the HTN associated with obesity is more likely to be Stage 1, although each patient is an individual. ${ }^{3}$

Pre-HTN is also seen in adolescents. The concept of pre-HTN made its way into the pediatric literature with the publication of the Fourth Report, and it has been defined as BP values that fall between the 90th and 95th percentiles on a consistent basis, or $>120 / 80$ for adolescents. Preventive strategies could be most likely to help here, and effective interventions for someone who is prehypertensive might allow for alleviation of manageable risk before sustained HTN arises. While there are no large-scale studies of preHTN in children and adolescents, it has been recently shown that adults with pre-HTN have adverse cardiometabolic risk, and by tending to the prehypertensive patient, some of that risk may be attenuated. ${ }^{5}$

In recent years, there has been more attention paid to assessing body mass index (BMI) in children and adolescents. A child is considered overweight when BMI is $>85$ th percentile and obese when BMI is $>95$ th percentile. The term metabolic syndrome refers to a cluster of risk factors for the development of cardiovascular disease and diabetes mellitus (DM) that include alterations in serum lipid levels, insulin resistance, obesity (especially central obesity), and HTN. There is no consensus as to what defines metabolic syndrome for children and adolescents, yet several findings are considered comorbidities in the context of obesity. There have been some modifications made in a definition of the National Cholesterol Education Program Adult Treatment Panel III criteria. These criteria when applied to children and adolescents would require at least three of the following for the diagnosis of metabolic syndrome: serum triglycerides $>95$ th percentile, high-density lipoprotein $<5$ th percentile, systolic BP or diastolic BP $>95$ th percentile, and impaired glucose tolerance. ${ }^{6}$

To date, the Fourth Report is the latest set of guidelines that may be used to evaluate an adolescent patient with elevated BP. In that report, one can find comprehensive guidance on the proper technique for BP measurement, tables to assess BP relative to age, gender and stature, and advice on the appropriate diagnostic interventions at the time of diagnosis. There is a clinical reference tool available as well that can be easily downloaded to use in clinical practice, and the link to that website is provided in the references. ${ }^{7}$

Another more recently published review by Flynn and Falkner discusses adolescent HTN in the context of obesity. They emphasize the need to pay attention to reduction of future cardiovascular risk. ${ }^{4}$

\section{Blood pressure variability, determination of the rate of progression from prehypertension to hypertension}

The issues of adolescent BP changes, HTN, and BP variability have been discussed for decades. In a review of the literature over the past quarter of a century, what is most striking is the number of different definitions of the concept of BP variability. In 1974, Kilcoyne et al noted changes in the "maturing circulation" of over 3500 high school students in Harlem. Mercury sphygmomanometers were used to measure $\mathrm{BP}$, and the values used to determine HTN were in line with current levels for adults (systolic 140, diastolic 90). They had interesting conclusions: "overall, contrary to traditional views, the development of HTN may begin in adolescence" and "methods of detection which appear satisfactory in adulthood are insufficient to uncover the true prevalence rates of HTN in this group." ${ }^{8}$ The same author concluded 
a few years later that there is a high degree of variability in BP in the adolescent population, making it all the more challenging to assess. ${ }^{9}$ Harshfield et al looked at a group of healthy, normotensive adolescents and measured BP with ambulatory BP monitoring (ABPM), and they concluded that race and gender are important in determining the changes that are observed between awake and asleep individuals. There is a diurnal variation in BP noted with ABPM use, and this displays that $\mathrm{BP}$ is an ever changing variable. ${ }^{10} \mathrm{As}$ more attention has been directed at targeting organ disease, there have also been attempts to link variability with evidence of target organ involvement. Belsha and colleagues looked at patterns of BP variability by way of ABPM while assessing markers of cardiac and renal abnormalities, and they concluded that adolescents with borderline and mild essential HTN and left ventricular hypertrophy had similar levels of fall in diurnal BP when compared to adolescents who were normotensive and to adolescents who were hypertensive but had normal left ventricular mass. ${ }^{11}$ The impact of birth weight is often discussed as a determining issue in the risk of development of HTN. Lurbe and colleagues assessed the relationship between birth weight and ambulatory $\mathrm{BP}$ variability by doing ABPM studies in over 600 healthy children from age 4 to 18 years. While not a completely adolescent population, the mean age was 9.9 years. They found a relationship between birth weight and what they called "BP variability," estimated as the standard deviation from discontinuous noninvasive BP monitoring techniques. They also felt that high BP variability was at least partially independent of BP values, and that there was need for further assessment of this phenomenon. ${ }^{12}$

With the publication of the Fourth Report in 2004, the concept of pre-HTN was introduced into the pediatric literature, and the definition is noted above. McNiece and colleagues did an extensive assessment of 6790 adolescents in the Houston, Texas school system. Utilizing oscillometric BP measuring devices, they performed a cross sectional assessment of BP and found that nearly $20 \%$ of those adolescents were at risk for HTN. In their initial screen, 81.1\% were normal, 9.5\% were classified as having pre-HTN, and 9.4\% had HTN. After three sets of readings, $81.1 \%$ were again normal, $15.7 \%$ had pre-HTN, and $3.2 \%$ had HTN. The authors explained a number of limitations of the study, and yet they also point to some of the issues that confront clinicians every day, including where to place a patient whose BP is in the normal range at one visit, but in the pre-HTN or HTN range at other times. ${ }^{13}$ Falkner and colleagues looked at the National Childhood Blood Pressure database to assess rates of progression from pre-HTN to HTN. In a group of over
8500 adolescents who had serial single BP measurements taken, they were able to determine that about $7 \%$ per year progressed from pre-HTN to HTN. This underscores that pre-HTN is predictive of HTN and that preventive strategies might be beneficial. ${ }^{14}$

There is evidence that BP measured in adolescence will predict future BP, and adolescents with BP levels in the higher portion of the $\mathrm{BP}$ distribution curve tend to maintain that position over time, a condition called tracking. It is estimated that there is an approximate incidence of adolescent HTN of 7\% per year. The data upon which this estimate is based have limitations as they are based upon a single measurement. If one looks among adolescents with pre-HTN and HTN combined, $68 \%$ of boys and $43 \%$ of girls had preHTN or HTN 2 years later. This raises the point that even a single elevated BP measurement in an adolescent raises the risk of having eventual persistent BP elevation. ${ }^{4}$

In recent years, $\mathrm{ABPM}$ has been increasingly used in adolescents. It may be useful for identifying isolated nocturnal HTN, characterizing BP patterns, and assessing therapeutic interventions. In adults, ABPM is felt to be superior to casual monitoring to predict cardiovascular morbidity and mortality, but the associations are less definitive in younger patients. Early evidence does seem to parallel adult data. In an article from 2008, Acosta and McNiece review the use of ABPM, discuss the limitations of the technology, and focus on indications for the use and interpretation of the data obtained in such a study. They point out that there is a need for true cardiovascular endpoints, as opposed to populationderived norms, to enhance the usefulness of this monitoring of $\mathrm{BP}$ in order to assess risks to patients. ABPM allows for a number of parameters to be assessed to establish risks of future cardiovascular outcomes, and thus it is a powerful tool that warrants more attention. ${ }^{15}$

It remains a practical challenge to the clinician to know exactly when to intervene with an elevated reading or set of readings. In addition to the $\mathrm{BP}$ values, one must take context into account. Such context includes the adolescent's personal history and examination, social history, and the family history of not only HTN, but also its consequences. Given the genetic and familial nature of this problem, one must involve the entire family in strategies that include lifestyle changes as well. Recently published recommendations on the use of ABPM in adolescents describe the usefulness of the technique, as well as what areas of the findings should be assessed. There was a clear call for the need for larger data sets of normative values, but it will take time for these to be collected. As ABPM becomes more widely accepted, 
further refinements of the use of this modality will become apparent. ${ }^{16}$

\section{Management Nonpharmacologic management}

Nonpharmacologic management is referred to as TLC in the Fourth Report. It is generally accepted that patients with preHTN, but without evidence of target organ disease, should be counseled in ways to effect TLC. In patients with diagnosed HTN, either Stage 1 or Stage 2, lifestyle changes should at least be used as adjuncts to therapy. ${ }^{3}$ A number of recent reviews of HTN that discuss therapy as part of the overall issue of HTN contribute to the advice that these nonpharmacologic interventions are important to incorporate into an overall treatment regimen for hypertensive patients. ${ }^{17-20}$ A very comprehensive review can also be found in a report published by the European Society of Hypertension. That report provides general recommendations for lifestyle measures that include: a prescribed amount of moderate to vigorous physical aerobic exercise; dietary guidance with respect to avoidance of excess sugar, soft drinks, saturated fat and salt; the implementation of behavioral changes that are tailored for the characteristics of not only the patient, but also the family; the provision of educational support and materials; establishment of realistic goals; development of a reward system; and recommendations regarding participation in competitive sports. ${ }^{21}$

\section{Lifestyle changes: general thoughts about weight loss and exercise}

The Fourth Report lists diet, exercise, and weight loss as potential lifestyle changes. A combination of diet modification, aerobic exercise, and weight loss consistently reduce $\mathrm{BP}$ and could therefore form the cornerstone for the initial intervention for obesity-induced HTN.

There are a number of strategies to treat obesity, and they include lifestyle changes, pharmacotherapy, and surgical interventions. Lifestyle changes, while difficult, are the initial steps to reducing weight, and the ways to do it are to reduce caloric intake and to exercise regularly. ${ }^{22}$

There have been both observational and interventional studies showing beneficial effects of weight loss in pediatric patients, and some of these have been reviewed in recent papers, ${ }^{23}$ yet there have been limited controlled trials. Rocchini and colleagues performed a randomized, controlled trial over a 20 week period, and three interventions were studied: diet alone, diet along with exercise, and a control group with no intervention at all. This study showed improvement in systolic BP from baseline in the intervention groups. ${ }^{24} \mathrm{~A}$ study like this can only address a limited aspect of the intervention in a defined period of time, and the long term effects of short term weight loss are unknown.

Weight loss not only reduces BP, but it may also improve some of the other cardiovascular risk factors such as dyslipidemia and insulin resistance that cluster with obesity. While weight loss is clearly a benefit to the patient, losing weight is generally a challenge for most patients and their families. Studies that have addressed weight reduction as a means of improving cardiovascular risk factors, including BP, have shown that interventions can be successful, yet adherence to these strategies is challenging, and these interventions tend to require a multidisciplinary approach. The intervention by a nutritionist is a key element to these programs, and some programs would also include psychological counseling as well as structured exercise programs. ${ }^{25,26}$ When HTN is affecting the obese child or adolescent, one cannot overlook that dealing with obesity is a part of the complex equation necessary for the optimal management of the patient's health. Another thing to consider when dealing with the child or adolescent with obesity is that it is often a family problem, and it will require commitment from the family to be successful.

Exercise training has also been shown to reduce BP for a limited period of time, on the order of 3-6 months, and once the exercise ends, it seems that BP returns to pretreatment levels. ${ }^{27,28}$

The forms of exercise felt to be most beneficial are aerobic activities such as running, brisk walking, swimming, or cycling, as opposed to static forms of exercise such as weightlifting. Some children may be participating in group activities in school physical education classes or in team sports, but they may need to increase the intensity of their involvement or the frequency at which they do these activities. While increasing these activities, attention should also be paid to reducing the amount of screen time in front of a television or computer that a child has. Clearly, these interventions are the safest and least prone to having adverse effects, but they remain challenging for families to pursue, and there is minimal evidence as well that these interventions are efficacious. ${ }^{3}$ It is also important to consider giving children and families some very concrete guidelines, rather than providing the general advice to "increase activity." Torrance and colleagues would suggest that children do 40 minutes of moderate to vigorous aerobic exercise three 
to five days per week. ${ }^{29}$ This could be a goal to strive for, yet it would certainly require a high degree of motivation on the part of the patient and also the patient's family.

\section{Lifestyle changes: dietary interventions}

It is very important to consider dietary strategies especially in the setting of obesity-associated HTN. If obesity is a cause or at least the primary contributor to HTN for the teenager, then one must tackle obesity as the underlying problem. There have been studies addressing dietary management of obesity-associated HTN, and even some in children. Of the various types of TLC, or nonpharmacologic management strategies, dietary interventions are ones that seem to be most often studied.

A number of nutrients have been examined such as sodium, potassium, calcium, folate, and caffeine; sodium has probably been the most extensively studied. While not every individual will be salt-sensitive, a modest sodium reduction would be beneficial, given the typical diet of most children and adolescents in the United States.

While dietary advice is recommended as first line therapy, there really is little evidence that it works. In a recent ${ }^{30}$ two year trial of potassium and calcium dietary supplementation in Chinese children who had salt-sensitive HTN, improvement in systolic BP was observed. Relative to obesity in general, there are many studies showing that diet, exercise and behavior modification can lead to improvement for children, however, there is also a high rate of recidivism.

When looking more specifically at younger patients with HTN, one can see that a few studies have looked at diet as a modifiable element of a young patient's life that can result in improvement. Moore et al looked at a group of children enrolled in the Framingham Heart Study, and they showed some beneficial effects on BP of a diet rich in fruits, vegetables and dairy products. ${ }^{31}$

The DASH (Dietary Approaches to Stop Hypertension) diet has been proven to lower BP primarily in adults, and some research in younger age groups using a similar plan has been done. The DASH diet goes beyond a low sodium diet and provides guidance for a diet rich in fruits and vegetables as well as low fat or nonfat dairy products. This diet is one that is low in sodium and enriched with potassium and calcium, and it also incorporates a higher intake of micronutrients, such as folate, and measures to reduce dietary fat intake. The reduction in dietary fat intake is important, given the likelihood for diets higher in fat content to promote weight gain as well as alterations in lipid levels. ${ }^{32}$
A study by Reinehr and colleagues looked at changes in weight and a number of cardiovascular risk factors to see if an intense intervention with a multidisciplinary approach could show improvement over a year. In addition to BP, other cardiovascular risk factors such as lipid profiles and measures of insulin resistance were studied. The intervention group had elements of physical exercise, nutritional education, and behavior therapy included, and the children who did have a drop in BMI also had a lower BP and improvement in the other measures of cardiovascular risk after a year. The authors did acknowledge some limitations to the study, including the fact that it was not randomized, so it may have been that the subjects (and their families) who were most successful may have been the more motivated ones to make a difference, and yet it does show that these strategies can work. ${ }^{25}$ An important message to take from this study is that the problem must be approached from a number of angles.

Couch and colleagues did a study that compared an intensive three-month intervention to a more routine type of nutritional intervention in adolescents referred to a tertiary care center HTN clinic and diagnosed with either pre-HTN or HTN. That study warrants some attention here. Two groups of children were studied over a three-month period. One group was called the DASH intervention. This group received extensive counseling as well as very close follow-up. This included a one-hour face-to-face counseling session between a dietician, the subject, and parent; a manual to take from the study center; eight weekly and two biweekly phone visits from a trained interventionist; and four biweekly mailings. The routine care group compared received a more standard dietary intervention, with the one-hour counseling session done in the clinic setting and provision of a booklet to take with them that basically discussed reduction of sodium intake, weight control by limiting high fat foods, reduction of portion control and eating nutrient dense forms of food. In addition to beneficial effects on BP, the DASH group also had significant changes in dietary intake of fruits, vegetables, and dairy products. ${ }^{33}$

A study by Gunther and colleagues in children and adolescents with DM and HTN explored the associations of the DASH diet in this population. It showed that children with type 1 DM following DASH guidelines had a markedly decreased chance of having HTN, but this was not observed in children with type 2 DM. In that study, the majority of subjects with type $2 \mathrm{DM}$ were obese. ${ }^{34}$ 


\section{Lifestyle changes: other elements to consider}

There are many reasons to counsel adolescents about the avoidance of tobacco, alcohol, and other drugs, and those issues have importance relative to the overall health of an individual and may improve BP control as well. The issue of stress on BP control has received some attention, and yet stress management techniques are not widely discussed with patients. In a review on stress management techniques in adolescents, Smith and Womack discuss the case of a teenager with what is described as mild HTN that was treated with Transcendental Meditation ${ }^{\circledR}$. That patient was normotensive at a 6-month follow-up interval. ${ }^{35} \mathrm{Saab}$ et al describe an interesting set of findings that a range of responses to behavioral stresses were found, with the adolescents with high BP being more vascularly responsive than those with labile BP. The adolescents with labile BP were more responsive than those with normal $\mathrm{BP} .{ }^{36}$ An interesting study of 35 adolescents with high normal BP, studied before the Fourth Report which defined pre-HTN in adolescents, randomized almost half of the group to a transcendental meditation intervention and the others to a health education control group. After two months, there was a statistically significant difference in resting systolic BP and other measures of cardiovascular reactivity. ${ }^{37}$

These techniques show usefulness in selected motivated patients, and they may be useful adjuncts to the overall treatment of adolescents with pre-HTN and HTN.

\section{Pharmacologic management}

The recommendations of the Task Force on Blood Pressure that are published in the Fourth Report remain the guiding principles upon which one decides about the initiation of pharmacologic management. There are a number of considerations to take into account when pharmacologic management is entertained. Long term consequences of untreated HTN are unknown, although it is generally felt that longstanding high BP is a risk factor for development of clinical problems at some point. There are also no data about the long term use of antihypertensive medications as well. There are limited indications recommended for use of medications, and they include patients who are symptomatic, those with secondary forms of HTN that need to be addressed in a specific way (for example kidney disease or coarctation of the aorta), those with evidence of target organ disease such as left ventricular hypertrophy, those with coincident diabetes (types 1 and 2), and those with persistent HTN despite nonpharmacologic treatment. That last category is particularly challenging, as it may be difficult to assess who has really taken the ideas and advice provided in the setting of nonpharmacologic treatment seriously. Exactly how long to allow the nonpharmacologic measures to work before initiating medication is also a challenge, and it seems more related to discussions one might have with the adolescent and his or her family. ${ }^{3}$

Once the decision has been made to initiate pharmacologic management, one should not completely abandon the nonpharmacologic measures; they should remain in place.

There have been some clinical trials including adolescents that have led to pediatric labeling of medications. These studies have been done primarily as single drug studies to provide safety, efficacy, and pharmacokinetic data. Frankly, all classes of antihypertensive medications have been shown to lower BP in this age group, yet there are not any specific recommendations on which medication to use in certain patients. There may be good reasons to consider using a certain class of medication in an adolescent with a specific underlying condition or comorbid condition. Such an example would be that of patients with DM, where one would consider the use of an angiotensin-converting enzyme inhibitor or angiotensin receptor blocker. This same set of medications would need to be used with caution in females of childbearing potential, as there may be risks to the developing fetus.

The published Fourth Report provides guidance on medications, including dosage and the potential for side effects. ${ }^{3}$ Another good source to become familiar with HTN management is the set of recommendations from the European Society of Hypertension published in 2009. ${ }^{21}$

\section{Conclusion}

When adolescents are identified with elevated BP, it may take some time before they are diagnosed with pre-HTN or HTN. In all adolescents, there ought to be discussions about strategies for healthy living, and in the context of elevated BP, one must include a number of strategies in the attempt to be successful at limiting cardiovascular morbidities in the long term. In many ways, detecting issues before they become more troublesome could lead to changes that have a longer and bigger impact. Not every intervention will work to the same extent or degree with every individual, yet by applying a number of strategies, there may be some opportunities for success. Also, treatment strategies should not be considered "either/or." Most, if not all, adolescent patients will benefit from strategies aimed at healthier habits of limited salt intake, a less sedentary lifestyle, and avoidance of tobacco products, excessive caffeine, and illicit drug use. Achieving that goal may require a number of different interventions and a high degree of motivation. 


\section{Disclosure/Acknowledgment}

Dr Batisky has funding support from the National Heart, Lung, and Blood Institute (NHLBI) [1 R01 HL098332-01A1, $\mathrm{M}$ Lande PI] to study the neurocognitive effects of HTN in children and adolescents.

\section{References}

1. Thomas CL, editor. Taber's Cyclopedic Medical Dictionary. 15th ed. Philadelphia: FA Davis Company; 1985.

2. Myetymology.com [homepage on the Internet]. c2008. Available from: http://www.myetymology.com/latin/adolescere.html. Accessed May 18, 2011.

3. The Fourth Report on the Diagnosis, Evaluation and Treatment of High Blood Pressure in Children and Adolescents. National High Blood Pressure Education Program Working Group on High Blood Pressure in Children and Adolescents. Pediatrics. 2004;114(2 Suppl 4th Report):555-576.

4. Flynn JT, Falkner BE. Obesity hypertension in adolescents: epidemiology, evaluation, and management. J Clin Hypertens. 2011;13(5):323-331.

5. Gupta AK, McGlone M, Greenway FL, Johnson WD. Prehypertension in disease-free adults: a marker for an adverse cardiometabolic risk profile. Hypertens Res. 2010;33(9):905-910.

6. Puri M, Flynn JT. Management of hypertension in children and adolescents with the metabolic syndrome. The Journal of Cardiometabolic Syndrome. 2006;1(4):259-268.

7. Clinical Reference Tool for the 4th Report on the Diagnosis, Evaluation, and Treatment of High Blood Pressure in Children and Adolescents. Available from: http://hp2010.nhlbihin.net/nhlbi_peds/hbppedpda. htm\#ppc. Accessed March 21, 2012.

8. Kilcoyne MM, Richter RW, Alsup PA. Adolescent hypertension. I. Detection and prevalence. Circulation. 1974;50(4):758-764.

9. Kilcoyne MM. Natural history of hypertension in adolescence. Pediatric Clinics of North America. 1978;25(1):47-53.

10. Harshfield GA, Alpert BS, Willey ES, Somes GW, Murphy JK, Dupaul LM. Race and gender influence ambulatory blood pressure patterns of adolescents. Hypertension. 1989;14(6):598-603.

11. Belsha CW, Wells TG, McNiece KL, Seib PM, Plummer JK, Berry PL. Influence of diurnal blood pressure variations on target organ abnormalities in adolescents with mild essential hypertension. Am J Hypertens. 1998;11(4 Pt 1):410-417.

12. Lurbe E, Torro I, Rodriguez C, Alvarez V, Redón J. Birth weight influences blood pressure values and variability in children and adolescents. Hypertension. 2001;38(3):389-393.

13. McNiece KL, Poffenberger TS, Turner JL, Franco KD, Sorof JM, Portman RJ. Prevalence of hypertension and prehypertension among adolescents. J Pediatr. 2007;150(6):640-644.

14. Falkner B, Gidding SS, Portman R, Rosner B. Blood pressure variability and classification of prehypertension and hypertension in adolescence. Pediatrics. 2008;122(2):238-242.

15. Acosta AA, McNiece KL. Ambulatory blood pressure monitoring: a versatile tool for evaluating and managing hypertension in children. Pediatr Nephrol. 2008;23(9):1399-1408.

16. Urbina E, Alpert B, Flynn J, et al; for American Heart Association Atherosclerosis, Hypertension, and Obesity in Youth Committee. Ambulatory blood pressure monitoring in children and adolescents: Recommendations for standard assessment. A scientific statement from the American Heart Association Atherosclerosis, Hypertension, and Obesity in Youth Committee of the Council on Cardiovascular Disease in the Young and the Council for High Blood Pressure Research. Hypertension. 2008;52(3):433-451.
17. Flynn JT. Hypertension in the young: epidemiology, sequelae and therapy. Nephrol Dial Transplant. 2009;24(2):370-375.

18. Lande MD, Flynn JT. Treatment of hypertension in children and adolescents. Pediatr Nephrol. 2009;24(10):1939-1949.

19. Feber J, Ahmed M. Hypertension in children: new trends and challenges. Clin Sci (Lond). 2010;119(4):151-161.

20. Brady TM, Feld LG. Pediatric Approach to Hypertension. Semin Nephrol. 2009;29(4):379-388.

21. Lurbe E, Cifkova R, Cruickshank JK, et al; for European Society of Hypertension. Management of high blood pressure in children and adolescents: recommendations of the European Society of Hypertension. J Hypertens. 2009;27(9):1719-1742.

22. Mathew B, Patel SB, Reams GP, Freeman RH, Spear RM, Villareal D. Obesity-hypertension: emerging concepts in pathophysiology and treatment. Am J Med Sci. 2007;334(1):23-30.

23. Sorof J, Daniels S. Obesity hypertension in children. Hypertension. 2002;40:441-447.

24. Rocchini AP, Karch V, Anderson J, et al. Blood pressure in obese adolescents: effect of weight loss. Pediatrics. 1988;82:16-23.

25. Reinehr T, de Sousa G, Toschke AM, Andler W. Long-term follow-up of cardiovascular disease risk factors in children after an obesity intervention. Am J Clin Nutr. 2006;84(3):490-496.

26. Denzer C, Reithofer A, Wabitsch M, Widhalm K. The outcome of childhood obesity management depends highly upon patient compliance. Eur J Pediatr. 2004;163(2):99-104.

27. Alpert BS. Exercise as a therapy to control hypertension in children. Int J Sports Med. 2000;21(Suppl 2):S94-S96.

28. Ribeiro MM, Silva AG, Santos NS, et al. Diet and exercise training restore blood pressure and vasodilatory responses during physiological maneuvers in obese children. Circulation. 2000;111:1915-1923.

29. Torrance B, McGuire KA, Lewanczuk R, et al. Overweight, physical activity and high blood pressure in children: a review of the literature. Vasc Health Risk Manag. 2007;3(1):139-149.

30. Mu JJ, Liu ZQ, Liu WM, et al. Reduction of blood pressure with calcium and potassium supplementation in children with salt sensitivity: a 2-year double-blinded placebo-controlled trial. J Hum Hypertens. 2005;19(6):479-483.

31. Moore LL, Singer MR, Bradlee ML, et al. Intake of fruits, vegetables, and dairy products in early childhood and subsequent blood pressure change. Epidemiology. 2005;16(1):4-11.

32. Sacks FM, Campos H. Dietary therapy in hypertension. N Engl J Med. 2010;362(22):2102-2112.

33. Couch SC, Saelens BE, Levin L, Dart K, Falciglia G, Daniels SR. The efficacy of a clinic-based behavioral nutrition intervention emphasizing a DASH-type diet for adolescents with elevated blood pressure. J Pediatr. 2008;152(5):494-501.

34. Gunther ALB, Liese AD, Bell RA, et al. Association between the dietary approaches to hypertension diet and hypertension in youth with diabetes mellitus. Hypertension. 2009;53(1):6-12.

35. Smith MS, Womack WM. Stress management techniques in childhood and adolescence: Relaxation training, meditation, hypnosis and biofeedback: Appropriate clinical applications. Clin Pediatr (Phila). 1987;26(11):581-585.

36. Saab PG, Llabre MM, Ma M, et al. Cardiovascular responsivity to stress in adolescents with and without persistently elevated blood pressure. J Hypertens. 2001;19(1):21-27.

37. Barnes VA, Treiber FA, Davis H. Impact of Transcendental Meditation $^{\circledR}$ on cardiovascular function at rest and during acute stress in adolescents with high normal blood pressure. J Psychosom Res. 2001;51(4):597-605. 


\section{Publish your work in this journal}

Adolescent Health, Medicine and Therapeutics is an international, peer-reviewed, open access journal focusing on health, pathology, and treatment issues specific to the adolescent age group. All aspects of health maintenance, preventative measures and disease treatment interventions are addressed within the journal and practitioners from all disciplines are invited to submit their work as well as healthcare researchers and patient support groups.. The manuscript management system is completely online and includes a very quick and fair peerreview system. Visit http://www.dovepress.com/testimonials.php to read real quotes from published authors.

Submit your manuscript here: http://www.dovepress.com/adolescent-health-medicine-and-therapeutics-journal 